ORIGINAL PAPER

\title{
SHORT-TERM PROGNOSIS OF CHILDHOOD HEPATOBLASTOMA IN RELATION TO ERCC1 C118T SINGLE NUCLEOTIDE POLYMORPHISM AND VEGF EXPRESSION
}

\author{
Yuan Wen*, Weiling Zhang*, Yi Zhang, Huimin Hu, Jing Li, Dongsheng Huang
}

${ }^{1}$ Department of Pediatrics, Beijing Tongren Hospital, Capital Medical University, Beijing, China

${ }^{*}$ Contributed equally.

To evaluate the short-term prognosis of pediatric hepatoblastoma (HB) in relation to excision repair cross-complementation gene 1 (ERCC1) C118T single nucleotide polymorphism (SNP) and VEGF expression.

ERCC1 C118T SNP and VEGF expression were detected and investigated in 31 children with $\mathrm{HB}$ undergoing platinum-based chemotherapy, to analyze their relationship with short-term pediatric $\mathrm{HB}$ prognosis.

CC $(38.7 \%$; 12/31), CT $(35.5 \%$; 11/31), and TT $(25.8 \% ; 8 / 31)$ ERCC1 C118T mutation types were identified. The Kaplan-Meier survival curve analysis showed that the CC group had a better short-term prognosis than the CT + TT group $(\mathrm{p}=0.010)$. VEGF was overexpressed in 14 cases $(45.2 \%)$ and underexpressed in 17 cases $(54.8 \%)$. The Kaplan-Meier survival curve analysis showed that the high VEGF expression group showed poorer short-term prognosis than the lower VEGF expression group $(\mathrm{p}=0.004)$.

In this study, ERCC1 C118T SNP in children with HB was mainly found to be mutant type CT + TT. Compared to wild type CC, children with the mutant type $\mathrm{CT}+\mathrm{TT}$ exhibited better treatment efficacy and remission with platinum-based chemotherapy as well as better survival rates. Moreover, the short-term prognosis of children with low VEGF expression was better than in those with high expression.

Key words: ERCC1 gene, single nucleotide polymorphism, VEGF, hepatoblastoma, prognosis, platinum drugs.

\section{Introduction}

Hepatoblastoma (HB) is a rare malignant tumor in children, accounting for less than $1 \%$ of all their malignancies. However, it remains the most common form of pediatric liver malignancy [1], the incidence rate of which is about 1.2-1.5 out of every million [2].

Before the early 1970s, HB treatment relied mainly on surgery, and the survival rate was only $20-30 \%$. With the introduction of platinum-based chemotherapeutic drugs (PDs), the post-treatment prognosis of $\mathrm{HB}$ has been greatly improved, and the 3-year overall survival rate can reach up to 60$70 \%$ [3, 4]. Platinum-based chemotherapeutic drugs are the main drugs used against HB. Almost all HB cases require preoperative and/or postoperative chemotherapy [5]. It has been reported that if well-differentiated PRETEXT Stage I epithelial HB can be completely resected, no postoperative chemotherapy 
is required, prognosis is good, and the survival rate can be $100 \%$ [6].

ERCC1 (excision repair cross-complementation gene 1) was the first DNA damage repair gene to be discovered in humans. It participates in the nucleotide excision repair (NER) process, which is an important mechanism in the resistance to PDs [7]. ERCC1 single nucleotide polymorphisms (SNPs), particularly the $\mathrm{C}-\mathrm{T}$ mutation at the $118^{\text {th }}$ codon in exon 4, can affect gene expression level and change gene copy number, thus affecting the resistance to PDs [8]. Many studies have reported that the ERCC1 C118T mutation exhibits a predictive value for PD efficacy and disease prognosis in different types of cancers, such as non-small cell lung cancer [8], ovarian cancer [9], gastric cancer [10], esophageal cancer [11], pancreatic cancer [12], and colorectal cancer [13]. Therefore, screening ERCC1 mutation before chemotherapy may have important guiding significance and prognostic value for chemotherapy.

Vascular endothelial growth factor (VEGF) is a highly-specific vascular endothelial cell mitogen that promotes endothelial cell proliferation, angiogenesis, and vascular permeability. It is the most important growth factor for angiogenesis [14]. Studies have shown that VEGF expression is closely related to angiogenesis, growth, invasion, and metastasis in tumors. High VEGF expression in liver tumors and childhood $\mathrm{HB}$ is also associated with poor prognosis $[15,16]$.

Since ERCC1-C118T mutation studies are rare and reported VEGF cases are limited in childhood HB, we used a retrospective cohort study to investigate the short-term prognosis of $\mathrm{HB}$ in children in relation to ERCC1 C118T mutation and VEGF expression. Here, ERCC1 C118T mutation and VEGF expression were detected in 31 children with $\mathrm{HB}$, and the effects of chemotherapy and short-term prognosis were reported for relation-based analysis and discussion.

\section{Materials and methods}

\section{Clinical data}

From January 2013 to June 2016, 45 children with $\mathrm{HB}$ (pathologically diagnosed and undergoing surgery) were treated at the Department of Pediatrics, Beijing Tongren Hospital, Capital Medical University. Among them, 31 children were tested for the ERCC1 C118T SNP using their blood samples and for VEGF expression from surgical samples of their tumors. The rest 14 patients were excluded due to refusal to sign informed consent. The children were staged according to the postoperative $\mathrm{HB}$ staging criteria developed by the Children's Oncology Group (COG; USA), and started on platinum-based chemotherapy regimens and follow-up routines. The follow-up endpoints were set either as relapse during follow-up/death or end of the 2-year follow-up. No case was lost during follow-up. This study was conducted in accordance with the declaration of Helsinki. This study was conducted with approval from the Ethics Committee of Capital Medical University. Written informed consent was obtained from all participants' guardians.

\section{Diagnostic and staging criteria}

Surgical specimens were obtained from the 31 children to confirm HB via pathological examination. The pathological classification criteria were based on the pathological types proposed by COG in 2014 [17], and all patients were classified based on the COG staging criteria (Table I) [18].

\section{Detection of C118T mutation}

Anticoagulant-containing peripheral blood samples $(2 \mathrm{ml})$ were taken from each child for DNA extraction using magnetic beads. DNA was extracted based on the instructions obtained from the Lab-Aid DNA Separation Kit (Zeesan Biotech Co. Ltd. Xiamen, China). DNA sequencing and TaqMan probing were used for detection and analysis.

\section{DNA sequencing method}

The ERCC1 primer sequences were as follows: F: GACCACAGGACACGCAGAC and R: TGAGGAACAGGGCACAGG. The amplified target gene fragment was a 3,162-3,595 bp long segmented sequence from the complete ERCC1 sequence. After amplification, the ABI 3730xL DNA sequencer (Hitachi, USA) was used for gene sequencing.

The TaqMan SNP Genotyping Assays Probe Kit (ABI) was customized to label VIC/FAM according to the human rs11615 (ASN118ASN; base sequence AAC118AAT). The Roche LightCycler ${ }^{\circledR} 480$ System was used to run PCR and the parameters were as follows: $50^{\circ} \mathrm{C}, 2 \mathrm{~min} ; 95^{\circ} \mathrm{C}, 10 \mathrm{~min} ; 95^{\circ} \mathrm{C}, 15 \mathrm{~s}$; and $60^{\circ} \mathrm{C}, 1 \mathrm{~min}$. The VIC and FAM fluorescent signals were then recorded and the Roche 480 SNP analysis software was used to determine whether the ERCC1 AAC118AAT site was homozygous or heterozygous.

Table I. Postoperative COG Staging criteria of HB

\begin{tabular}{lc}
\hline StAGING & Definition \\
\hline Stage I & Signifies negative surgical margins \\
\hline Stage II & $\begin{array}{c}\text { Has residual microscopic disease } \\
\text { at the margins }\end{array}$ \\
\hline Stage III & $\begin{array}{c}\text { Represents grossly positive margins } \\
\text { or regional lymph node involvement }\end{array}$ \\
\hline Stage IV & Indicates metastatic disease \\
\hline
\end{tabular}




\section{Detection of VEGF}

Immunohistochemical staining was used to detect VEGF expression in the surgical/liver biopsy specimens. The human VEGF immunohistochemical staining kit (Yaji Co. Ltd., Shanghai, China) was used as per the manufacturer's instructions. The general steps were as follows: paraffin embedding, tissue slicing ( $3 \mu \mathrm{m}$ thickness), slice baking, dewaxing, hydration, antigen retrieval, sequential incubation and washing with primary and secondary antibodies, coloration with DBA solution, dehydration, hyalinization, mounting, and observation. The positive control was obtained from Yaji Co. Ltd., and the blank control involved washing with PBS solution in place of the primary antibodies.

Currently, no standard methodologies exist to judge VEGF immunohistochemical staining results in children with HB. After referring to other studies and comprehensively considering the strength of staining and the number of colored cells in this study, yellow- or brown-yellow-stained granules in the cytoplasm were defined positive cell staining. All the slices were observed under a $400 \times$ microscope with 5 randomly selected fields from each slice. The proportion of positively-stained cells in the fields was averaged and scored as follows: proportion $\leq 5 \%, 0$ points; $6-25 \%, 1$ point; $26-50 \%, 2$ points; $51-75 \%, 3$ points; and $\geq 75 \%, 4$ points; for staining intensity: no stain, 0 points; light yellow, 1 point; yellow, 2 points; and brown, 3 points. The total score was then calculated as a product of positive cell proportion and staining intensity giving the following results: 0-4 points, low expression; and 5-12 points, high expression [19].

\section{Efficacy analysis and follow-up}

Treatment efficacy was determined using the following parameters based on the RECIST 1.1 evaluation criteria [20]:

1. Complete response (CR): Disappearance of all target lesions. Any pathological lymph nodes (whether target or non-target) must have their short axis reduced to $<10 \mathrm{~mm}$.

2. Partial response (PR): At least a 30\% decrease in the sum of the diameters of the target lesions, using the baseline sum diameters as a reference.

3. Progressive disease (PD): At least a $20 \%$ increase in the sum of the diameters of the target lesions, using the smallest sum value in the study as a reference (this includes the baseline sum if it is the smallest sum value in the study). In addition to the $20 \%$ relative increase, the sum value must also demonstrate an absolute increase of at least $5 \mathrm{~mm}$. The appearance of one or more new lesions is also considered to be progression.

4. Stable disease (SD): Neither sufficient shrinkage to qualify for PR, nor sufficient increase to qualify for $\mathrm{PD}$, using the smallest sum diameter value in the study as a reference.

Follow-up started at the end of chemotherapy and lasted for 2 years. The end point was decided as recurrence/death during the follow-up or completion of the 2-year follow-up.

At the end of the study, since there were no SD patients, the $\mathrm{CR}$ and $\mathrm{PR}$ cases were categorized into the remission group (Group R), and the PD, relapse, and death cases were categorized into the non-remission group (Group NR). Group R was considered to be progression-free survival (PFS) cases [20].

\section{Statistical analysis}

The SPSS 20.0 software was used for statistical analysis. Count data was determined using Fisher's exact test or continuous calibration $\chi^{2}$ test. Survival analysis was performed using the Kaplan-Meier method, and $\mathrm{p}<0.05$ was considered to be statistically significant.

\section{Results}

\section{General information}

Thirty-one cases were included in the study. Diagnosis age ranged from 1 month to 156 months, with the average being $34.23 \pm 34.89$ months. The general information is shown in Table II. The number of male and female patients were 22 and 9, respectively. There were 2 stage II, 12 stage III, and 17 stage IV cases. The pathological types included 20 epithelial HB and 11 mixed HB cases. The $\alpha$-fetoprotein (AFP) levels in all the patients were more than $1000 \mathrm{ng} / \mathrm{ml}$.

\section{Chemotherapy drugs and follow-up}

All 31 patients underwent chemotherapy regimens of platinum-based drugs combined with other drugs.

Table II. General information of 31 enrolled children

\begin{tabular}{lcccccccc}
\hline & M & F & Stage II & Stage III & Stage IV & $\begin{array}{c}\text { Epithelial } \\
\text { HB }\end{array}$ & Mixed HB \\
\hline $\mathrm{n}$ & 22 & 9 & 2 & 12 & 17 & 20 & 11 \\
\hline Ratio & $71.0 \%$ & $29.0 \%$ & $6.5 \%$ & $38.7 \%$ & $54.8 \%$ & $64.5 \%$ & $35.5 \%$ \\
\hline
\end{tabular}


The patients were divided into low-risk, intermediate-risk, and high-risk based on the COG risk stratification [21]. Platinum-based C5V (low risk) and C5VD (intermediate and high risk) treatments were used for the different risk stratifications [21]. The C5V regimen consisted of cisplatin $\left(90 \mathrm{mg} / \mathrm{m}^{2}\right.$, $>1$ year old; or $3 \mathrm{mg} / \mathrm{kg},<1$ year old) on day 1 , and vincristine $\left(1.5 \mathrm{mg} / \mathrm{m}^{2}\right.$ with a maximum dose of $2 \mathrm{mg}$ ) and 5-fluorouracil $\left(600 \mathrm{mg} / \mathrm{m}^{2}\right)$ on day 2. The C5VD regimen consisted of cisplatin (90 $\mathrm{mg} / \mathrm{m}^{2},>1$ year old; or $3 \mathrm{mg} / \mathrm{kg},<1$ year old) on day 1 , vincristine $\left(1.5 \mathrm{mg} / \mathrm{m}^{2}\right.$ with a maximum dose of $2 \mathrm{mg}$ ) and 5-fluorouracil $\left(600 \mathrm{mg} / \mathrm{m}^{2}\right)$

\section{A}

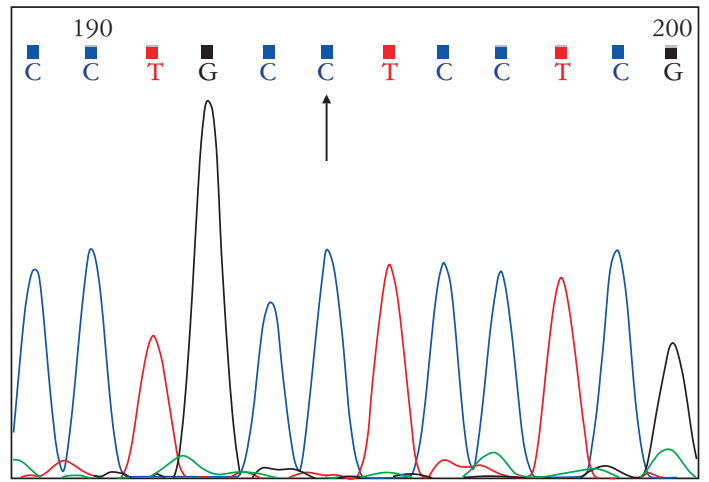

B

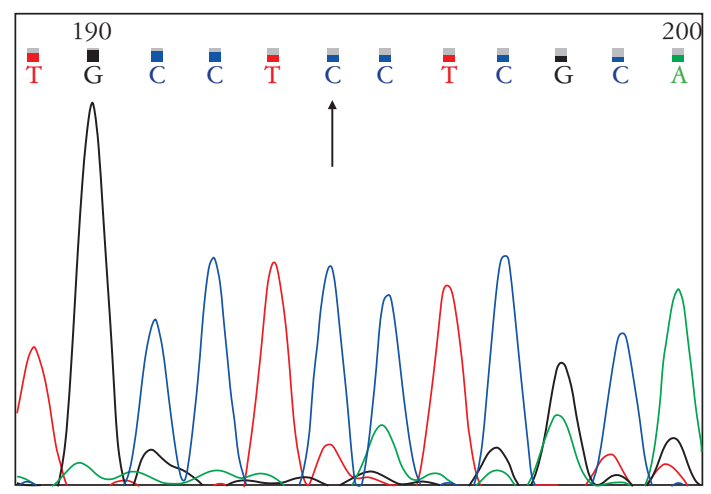

C

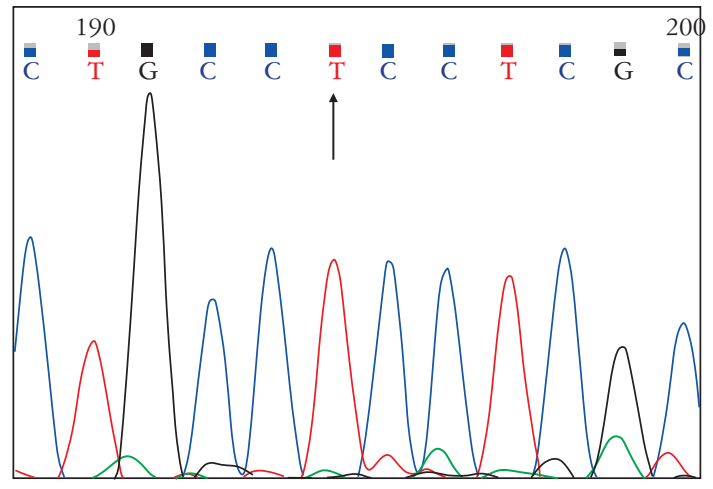

Fig. 1. Examples of ERCC1 gene C118T mutation: A) CC wild homozygote; B) CT mutant heterozygote; C) TT mutant homozygote on day 2 , and doxorubicin $\left(25 \mathrm{mg} /\left(\mathrm{m}^{2} \cdot \mathrm{d}\right)\right)$ on days 2 and 3 .

At the end of the follow-up, $38.7 \%$ of the patients (12/31) achieved CR, 19.4\% (6/31) achieved PR, $9.7 \%$ (3/31) achieved PD, 19.4\% (6/31) relapsed, and $12.9 \%$ (4/31) died. Group R accounted for $58.1 \%$ $(18 / 31)$ of the patients and Group NR accounted for $41.9 \%(13 / 31)$.

\section{Detection of C118T mutation}

The analysis of the ERCC1 C118T mutation in the 31 patients detected $38.7 \%(12 / 31)$ as CC wild homozygote, $35.5 \%(11 / 31)$ as CT mutant heterozygote, and $25.8 \%(8 / 31)$ as TT mutant homozygote. Mutant type CT + TT accounted for $61.3 \%$ of all patients, and wild type CC accounted for $38.7 \%$ of all patients. The results of the gene sequencing are shown in Fig. 1 (the arrow shows the ERCC1 SNP location on exon 4 codon 118). Statistical significance of deviations from the Hardy-Weinberg equilibrium was determined using the Pearson's chi-square test $\left(\chi^{2}=1.083, \mathrm{p}=0.582\right)$, which meant that the distribution of the C118T polymorphism was within the Hardy-Weinberg equilibrium.

\section{VEGF test results}

VEGF detection in the pathological specimens of the 31 patients showed high VEGF expression in $45.2 \%(14 / 31)$ and low VEGF expression in $54.8 \%$ $(17 / 31)$ of the specimens.

\section{Relationship between clinical stage and VEGF expression}

Patients were divided into the II + III stage group (Group II + III) and the IV stage group (Group IV) based on the HB clinical staging, and into the high expression (Group $\mathrm{H}$ ) and low expression (Group L) groups using Fisher's exact test based on the VEGF detection results (Table III). The ratio of high VEGF expression in Group II + III was observed to be 3/14 and Group IV was $11 / 17$. The difference was statistically significant $(\mathrm{p}=0.029)$. Moreover, the high VEGF expression rate in Group II + III was lower than that of Group IV.

\section{Relationship between VEGF expression and prognosis}

The results of the Fisher's exact test after the 2-year follow-up revealed that PFS proportion was $28.6 \%$ in Group H (4/14) and 82.4\% in Group L (14/17). The difference was statistically significant $(\mathrm{p}=0.004$; Table IV).

The results of the Kaplan-Meier survival analysis (Fig. 2) suggested that the PFS status in Group L was better than that in Group H. The difference was statistically significant $(\mathrm{p}=0.005)$. 
Table III. Relationship between clinical stage and VEGF expression in $\mathrm{HB}$

\begin{tabular}{lccc}
\hline & Group II + III & Group IV & SuM \\
\hline Group H & 3 & 11 & 14 \\
\hline Group L & 11 & 6 & 17 \\
\hline Sum & 14 & 17 & 31 \\
\hline
\end{tabular}

Table IV. VEGF expression and prognosis of $\mathrm{HB}$

\begin{tabular}{lccc}
\hline & Group H & Group L & SUM \\
\hline Group R & 4 & 14 & 18 \\
\hline Group R/D & 10 & 3 & 13 \\
\hline Sum & 14 & 17 & 31 \\
\hline
\end{tabular}

Table V. ERCC1 C118T types with prognosis of children with $\mathrm{HB}$

\begin{tabular}{lccc}
\hline & TT + CT & CC & SuM \\
\hline Group R & 16 & 2 & 18 \\
\hline Group R/D & 5 & 8 & 13 \\
\hline Sum & 21 & 10 & 31 \\
\hline
\end{tabular}

Relationship between the ERCC1 C118T types and prognosis

Patients were divided into mutant TT $+\mathrm{CT}$ and wild CC groups based on the ERCC1 C118T types. The continuous calibration chi-square test (Table V) suggested that the 2-year PFS ratio was $76.2 \%$ in group TT + CT (16/21) and $20 \%$ in group CC $(2 / 10)$. The difference was statistically significant $(\mathrm{p}=0.010)$ i.e., the post-treatment PFS rate in Group TT + CT was higher than that in Group CC.

The Kaplan-Meier analysis revealed that the survival condition for the 2-year PFS in group TT $+\mathrm{CT}$ was better than that in group CC (Fig. 3). The difference was statistically significant ( $p=0.008)$.

\section{Discussion}

Currently, HB therapeutic strategies mainly consist of surgery and chemotherapy. The surgical removal of lesions has a crucial influence on prognosis [22]. In children who cannot undergo complete resection, prognosis mainly depends on chemotherapy. While preoperative adjuvant chemotherapy can increase the rate of surgical resection, Postoperative chemotherapy is of great significance in eliminating residual lesions $[23,24]$. The application of PDs in the 1970s significantly increased the prognosis of HB treatments. Currently, cisplatin alone (lowdose chemotherapy regimen) for low-risk $\mathrm{HB}$, PLADO and C5V for standard-risk HB, and Super

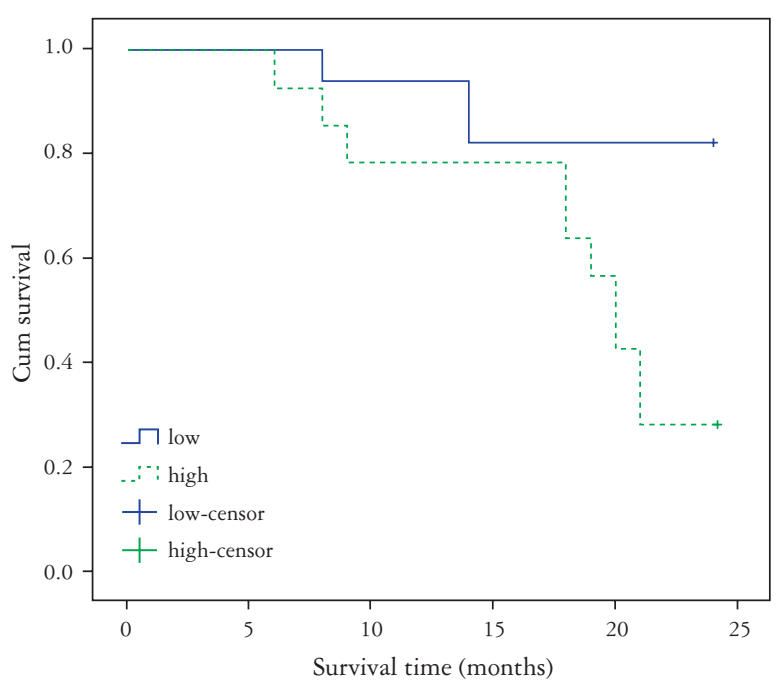

Fig. 2. Kaplan-Meier 2-year progression free survival analysis of VEGF high expression group and low expression group

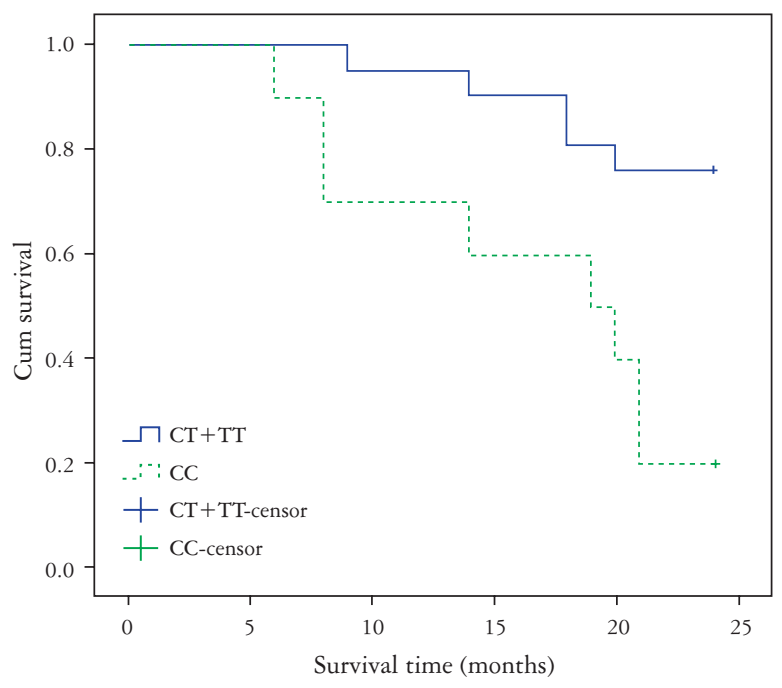

Fig. 3. Kaplan-Meier 2-year progression free survival analysis of ERCC1 gene CC group and CT+TT group

PLADO and C5VD for high-risk HB are the main, indispensable platinum drugs used for treatment $[22$, 25, 26]. After entering the body, the platinum drugs bind to DNA to form intra-strand and inter-strand crosslinks, which induces DNA damage due to this covalent DNA binding, thereby inhibiting DNA synthesis and replication, causing cell death, and suppressing tumor cell growth [27]. DNA damage correction caused by platinum drugs in vivo mainly depends on the nucleotide excision/repair mechanism, which specifically removes the platinum-DNA adducts, repairs DNA damage, and causes PD resistance [28].

ERCC1 was the first DNA damage repair gene to be discovered in humans, and its expressed product participates in DNA strand cleavage and damage 
repair via the NER pathway [29]. It is the key rate-limiting enzyme in the nucleotide excision/ repair mechanism [30]. A common and significant ERCC1 SNP is the $\mathrm{C} \rightarrow \mathrm{T}$ mutation at codon 118 on exon 4, causing the codon AAC to become AAT [13]. This is a nonsense mutation, which could consist of a $\mathrm{C} / \mathrm{C}$ wild homozygote, $\mathrm{T} / \mathrm{T}$ mutant homozygote, or $\mathrm{C} / \mathrm{T}$ mutant heterozygote. There is no change in the encoded aspartic acid, but the usage frequency of the AAT codon is lower than that of AAC, resulting in the decreased expression of the mutant ERCC1 gene carrying the $T$ base [31]. Higher ERCC1 expression levels are associated with stronger NER abilities and higher resistance to platinum drugs [32]. C118T mutation reduces ERCC1 expression, NER, and the ability of ERCC1 to repair DNA damage caused by platinum. Patients with the $\mathrm{T}$ allele may also be more sensitive to PDs [31, 32]. Globally, many studies in non-small cell lung cancer [8], ovarian cancer [9], gastric cancer [10], esophageal cancer [11], pancreatic cancer [12], and colorectal cancer [13] have shown patients with the CT or TT genotypes in ERCC1 C118T mutation to be more sensitive to PDs when compared to patients with the CC genotype.

In this study, the ERCC1 C118T SNP in the 31 children with $\mathrm{HB}$ was mainly found to be the $\mathrm{CT}+$ TT (19/31) mutation $(61.3 \%$ of the children exhibited the $\mathrm{C} \rightarrow \mathrm{T}$ mutation). This study further analyzed the correlation between the ERCC1 genotype and relapse/remission. The results showed that the remission rate in children with CT + TT was higher than those with CC. The 2-year survival analysis showed that the survival condition of group CT + TT was better than that of group CC i.e., the 2-year remission rate was high and survival condition was better. It is not difficult to infer that the children with CT + TT had a reduced ability to repair nucleotide excision; therefore, they had a lower ability to repair PD-induced damage, were more sensitive to PDs, and exhibited better chemotherapy results. The above inference is consistent with the reports of other studies both in and outside China [8-13].

VEGF is a highly-specific vascular endothelial cell mitogen that promotes vascular endothelial cell proliferation, angiogenesis, and increases vascular permeability [14]. It is the most important cytokine in tumor angiogenesis. VEGF is closely related to the growth, invasion, metastasis, and prognosis of tumors $[15,16]$. In recent years, studies have shown that pediatric liver malignancies have the characteristics of wide-range angiogenesis, and new blood vessels cause rapid tumor growth and lead to poor prognosis [16].

This study showed that VEGF expression rate was significantly higher in clinical stage IV patients (distant tumor metastasis) when compared to clinical stage II + III patients. This suggested that VEGF may play certain roles in tumor invasion and metastasis. Moreover, the 2-year remission rate was significantly higher and survival was better in children with low VEGF expression when compared to those with high VEGF expression. This suggested that VEGF had a certain predictive effect on the prognosis of such children. Recently, experiments have shown that the application of the VEGF antibody can significantly inhibit the angiogenesis and tumor growth of human $\mathrm{HB}$ cells cultured in vitro [33]. VEGF-targeted drugs, such as the VEGF monoclonal antibody bevacizumab and the VEGF receptor-selective tyrosine kinase inhibitor sorafenib, developed for malignant tumors and approved by the FDA, have demonstrated efficacy in treating kidney and liver cancers [34, 35]. Bevacizumab combined with sorafenib was also reported to stabilize $\mathrm{HB}$ and reduce $\alpha$-fetoprotein levels in a patient suffering from recurrent $\mathrm{HB}$ and distant metastasis [36]. Combined with the results from the present study, results showing patients with distant $\mathrm{HB}$ metastasis (stage IV) having high VEGF expression and patients with high VEGF expression having low 2-year chemotherapy remission rates suggested VEGF-targeted treatments as good alternative treatment options against $\mathrm{HB}$ in children with high VEGF expression.

In this study, ERCC1 C118T SNP in children with $\mathrm{HB}$ was mainly found to consist of the CT + TT mutant type. Children with $\mathrm{T}$ allele mutations had better treatment efficacy and higher 2-year PFS rates. Moreover, stage IV patients were found to have high VEGF expression rates and consequently, low 2-year PFS rates.

However, this study was limited as it was a single-center trial. Additionally, only a few cases were included and the study time was short. In order to analyze medium/long-term survival conditions, multi-center trials, larger sample sizes, and longer study times are required.

This research was supported by Beijing Yizhuang Economic and Technological Development Zone Leading Talent Program \{(2017)-8\}.

The authors declare no conflict of interest.

\section{References}

1. Czauderna P, Lopezterrada D, Hiyama E, et al. Hepatoblastoma state of the art: pathology, genetics, risk stratification, and chemotherapy. Curr Opin Pediatr 2014; 26: 19-28.

2. Allan BJ, Parikh PP, Diaz S, et al. Predictors of survival and incidence of hepatoblastoma in the paediatric population. HPB (Oxford) 2013; 15: 741-746.

3. Hiyama E, Hishiki T, Watanabe $\mathrm{K}$, et al. Mortality and morbidity in primarily resected hepatoblastomas in Japan: Experience of the JPLT (Japanese Study Group for Pediatric Liver Tumor) trials. J Pediatr Surg 2015; 50: 2098-2101.

4. Brown J, Perilongo G, Shafford E, et al. Pretreatment prognostic factors for children with hepatoblastoma - results from 
the International Society of Paediatric Oncology (SIOP) study SIOPEL 1. Eur J Cancer 2000; 36: 1418-1425.

5. Ismail H, Broniszczak D, Kaliciński $\mathrm{P}$, et al. Changing treatment and outcome of children with hepatoblastoma: analysis of a single center experience over the last 20 years. J Pediatr Surg 2012; 47: 1331-1339.

6. Malogolowkin MH, Katzenstein HM, Meyers RL, et al. Complete Surgical Resection Is Curative for Children With Hepatoblastoma With Pure Fetal Histology: A Report From the Children's Oncology Group. J Clin Oncol 2011; 29: 3301-3306.

7. Park CH, Bessho T, Matsunaga T, et al. Purification and characterization of the XPF-ERCC1 complex of human DNA repair excision nuclease. J Biol Chem 1995; 270: 22657-22660.

8. Deng Q, Yang H, Lin Y, et al. Prognostic value of ERCC1 mRNA expression in non-small cell lung cancer, breast cancer, and gastric cancer in patients from Southern China. Int J Clin Exp Pathol 2014; 7: 8312-8321.

9. Xu Q, Ding YY, Song LX, et al. Correlation of UGT1A1 and ERCC1 gene polymorphisms with the outcome of combined irinotecan plus cisplatin treatment in recurrent ovarian cancer. Genet Mol Res 2015; 14: 7241-7247.

10. Zhang YY, Gu KS, Wu HY, et al. Correlation of ERCC1 expression in peripheral blood lymphocytes with outcomes of patients with gastric cancer treated with oxaliplatin-based adjuvant chemotherapy. Genet Mol Res 2015; 14: 15921-15929.

11. Yu X, Xiao H, Zhao B, et al. DNA repair gene ERCC1 C118T polymorphism predicts sensitivity of recurrent esophageal cancer to radiochemotherapy in a Chinese population. Thorac Cancer 2015; 6: 741-748.

12. He MG, Zheng K, Tan D, et al. Association between ERCC1 and ERCC2 gene polymorphisms and susceptibility to pancreatic cancer. Genet Mol Res 2016; 15: gmr7879.

13. Viguier J, Boige V, Miquel C, et al. ERCC1 codon 118 polymorphism is a predictive factor for the tumor response to oxaliplatin/5-fluorouracil combination chemotherapy in patients with advanced colorectal cancer. Clin Cancer Res 2005; 11: 6212-6217.

14. Costache MI, Ioana M, Iordache S, et al. VEGF Expression in Pancreatic Cancer and Other Malignancies: A Review of the Literature. Rom J Intern Med 2015; 53: 199-208.

15. Hsieh MC, Hsu HT, Hsiao PC, et al. Role of VEGF-C Gene Polymorphisms in Susceptibility to Hepatocellular Carcinoma and Its Pathological Development. J Clin Lab Anal 2014; 28: 237-244.

16. Sun XY, Wu ZD, Liao XF, et al. Tumor angiogenesis and its clinical significance in pediatric malignant liver tumor. World J Gastroenterol 2005; 11: 741-743.

17. Aronson DC, Meyers RL. Malignant tumors of the liver in children. Semin Pediatr Surg 2016; 25: 265-275.

18. Kapadia RN, Zeidman MJ, Burjonrappa S. Advanced Hepatoblastoma: A Review of Current Management Strategies. Adv Res Gastroentero Hepatol 2015; 1: 555551.

19. Cai S, Han K. Research on expression and importance of $\mathrm{p} 53$, p16 and VEGF-C in cervical cancer. J Gynecol Obstet Biol Reprod (Paris) 2015; 44: 639-645.

20. Eisenhauer EA, Therasse P, Bogaerts J, et al. New response evaluation criteria in solid tumours: revised RECIST guideline (version 1.1). Eur J Cancer 2009; 45: 228-247.

21. Kremer N, Walther AE, Tiao GM. Management of hepatoblastoma: an update. Curr Opin Pediatr 2014; 26: 362-369.

22. Hiyama E. Pediatric hepatoblastoma: diagnosis and treatment. Transl Pediatr 2014; 3: 293-299.

23. Czauderna P. Hepatoblastoma throughout SIOPEL trials - clinical lessons learnt. Front Biosci (Elite Ed) 2012; 4: 470-479.

24. Perilongo G, Malogolowkin M, Feusner J. Hepatoblastoma clinical research: lessons learned and future challenges. Pediatr Blood Cancer 2012; 59: 818-821.

25. von Schweinitz D. Hepatoblastoma: recent developments in research and treatment. Semin Pediatr Surg 2012; 21: 21-30.
26. Ortega JA, Douglass EC, Feusner JH, et al. Randomized comparison of cisplatin/vincristine/fluorouracil and cisplatin/ continuous infusion doxorubicin for treatment of pediatric hepatoblastoma: A report from the Children's Cancer Group and the Pediatric Oncology Group. J Clin Oncol 2000; 18: 2665-2675

27. Dasari S, Tchounwou PB. Cisplatin in cancer therapy: molecular mechanisms of action. Eur J Pharmacol 2014; 740: 364-378.

28. Usanova S, Piée-Staffa A, Sied U, et al. Cisplatin sensitivity of testis tumour cells is due to deficiency in interstrand-crosslink repair and low ERCC1-XPF expression. Mol Cancer 2010; 9: 475-489.

29. Gazdar AF. DNA repair and survival in lung cancer - the two faces of Janus. N Engl J Med 2007; 356: 771-773.

30. Sancar A, Reardon JT. Nucleotide excision repair in E. coli and man. Adv Protein Chem 2004; 69: 43-71.

31. Yanlong Y, Lei X. The association between the ERCC1/2 polymorphisms and the clinical outcomes of the platinum-based chemotherapy in non-small cell lung cancer (NSCLC): a systematic review and meta-analysis. Tumour Biol 2014; 35: 2905-2921.

32. Yin M, Yan J, Martinez-Balibrea E, et al. ERCC1 and ERCC2 polymorphisms predict clinical outcomes of oxaliplatin-based chemotherapies in gastric and colorectal cancer: a systemic review and meta-analysis. Clin Cancer Res 2011; 17: 1632-1640.

33. Mccrudden KW, Hopkins B, Frischer J, et al. Anti-VEGF antibody in experimental hepatoblastoma: suppression of tumor growth and altered angiogenesis. J Pediatr Surg 2003; 38: 308-314.

34. Dorff TB, Longmate JA, Pal SK, et al. Bevacizumab alone or in combination with TRC105 for patients with refractory metastatic renal cell cancer. Cancer 2017; 123: 4566-4573.

35. Diaz-Beveridge R, Bruixola G, Lorente D, et al. An internally validated new clinical and inflammation-based prognostic score for patients with advanced hepatocellular carcinoma treated with sorafenib. Clin Transl Oncol 2018; 20: 322-329.

36. Marsh AM, Lo L, Cohen RA, et al. Sorafenib and bevacizumab for recurrent metastatic hepatoblastoma: stable radiographic disease with decreased AFP. Pediatr Blood Cancer 2012; 59: 939-940.

\section{Address for correspondence}

\section{Dongsheng Huang}

Department of Pediatrics

Beijing Tongren Hospital, Capital Medical University

No. 1 Dongjiaominxiang Dongcheng District

Beijing 100176, China

tel. +861058266032

$\mathrm{fax}+861058266032$

e-mail: cndongshenghuang@126.com 